\title{
The Mitochondrial Calcium Uniporter participates in Ischemia/Reperfusion Injury and in Cardioprotection by Ischemic Preconditioning
}

\author{
S-Z. Zhang ${ }^{1}$, Q. Xia ${ }^{1}$, C-M. Cao ${ }^{1}$, Q. Gao ${ }^{1}$, I.C. Bruce ${ }^{2}$ \\ ${ }^{1}$ Department of Physiology, Zhejiang University School of Medicine, Hangzhou, China \\ ${ }^{2}$ Department of Physiology, University of Hong Kong, Hong Kong, China \\ Email: xiaqiang@zju.edu.cn
}

\begin{abstract}
The objective of the present study was to determine whether the mitochondrial calcium uniporter plays a role in cardioprotection by ischemic preconditioning (IPC). Isolated rat hearts were subjected to $30 \mathrm{~min}$ regional ischemia by ligation of the left anterior descending artery followed by 120 min reperfusion. We found that both IPC and inhibition of the mitochondrial calcium uniporter during reperfusion improved recovery of left ventricular developed pressure, maximal rise velocity and end-diastolic pressure, and reduced infarct size and lactate dehydrogenase release. These protective effects were attenuated by activating the mitochondrial calcium uniporter. We conclude that the mitochondrial calcium uniporter is involved in the cardioprotection of ischemic preconditioning.
\end{abstract}

Keywords - Heart, mitochondrial calcium uniporter, ischemic preconditioning

\section{INTRODUCTION}

Ischemic preconditioning (IPC), which was originally identified by Murry and co-workers [1], provides powerful protection to the ischemic myocardium. Although various substances and signaling pathways may be involved in IPC [2], the precise mechanisms for its cardioprotective action remain elusive. Intensive studies showed that prolonged ischemia results in increased intracellular calcium concentration $[3 ; 4]$ and this calcium overload was thought to play a pivotal role in ischemia-reperfusion injury. It has been demonstrated that IPC can lower both intracellular and mitochondrial calcium during ischemia [5]. During myocardial performance, mitochondria accumulate significant amounts of calcium from the cytosol through the mitochondrial calcium uniporter, a highly selective calcium ion channel [6]. We hypothesized that the mitochondrial calcium uniporter may participate in the cardioprotection conferred by IPC. To test this hypothesis, we examined the effects of blockade and activation of the uniporter on the cardioprotective effect of IPC as measured by ventricular performance, infarct size and lactate dehydrogenase release.

\section{MethodOLOGY}

1) Langendorff heart preparation: Male SpragueDawley rats $(200 \pm 10 \mathrm{~g})$ were sacrificed by stunning and cervical dislocation. The hearts were excised and immediately placed in ice-cold Krebs-Henseleit (K-H) bicarbonate buffer containing (mmol/L): $\mathrm{NaCl} 118, \mathrm{KCl} 4.7$, $\mathrm{KH}_{2} \mathrm{PO}_{4} 1, \mathrm{NaHCO}_{3} 2.5, \mathrm{MgSO}_{4} 1.2, \mathrm{CaCl}_{2} 1.4$, glucose 10 . The hearts were then mounted to the Langendorff apparatus and perfused retrogradely with $\mathrm{K}-\mathrm{H}$ buffer gassed with $95 \% \mathrm{O}_{2}-5 \% \mathrm{CO}_{2}$ equilibrated at $\mathrm{pH} 7.3-7.4$ and $37^{\circ} \mathrm{C}$. $\mathrm{A}$ water-filled latex balloon was inserted into the left ventricle through the left atrium and the pressure was continuously monitored by a pressure transducer connected to the balloon. The initial value of end-diastolic pressure was set to 5-8 $\mathrm{mmHg}$ by adjusting the volume of the balloon. The parameters measured were left ventricular developed pressure (LVDP), left ventricular end-diastolic pressure (LVEDP) and maximal rise velocity ( $+\mathrm{dP} / \mathrm{dtmax})$.

2) Perfusion protocol of isolated heart: All isolated hearts received $30 \mathrm{~min}$ regional ischemia by ligation of the left anterior descending artery followed by $120 \mathrm{~min}$ reperfusion. The hearts in the IPC group experienced two IPC episodes: 5-min brief global ischemia and 5-min reperfusion, followed by sustained ischemia and reperfusion. Ruthenium red $\left(5 \times 10^{-6} \mathrm{M}\right)$, a blocker of the mitochondrial calcium uniporter, or spermine $\left(20 \times 10^{-6} \mathrm{M}\right)$, an activator of mitochondrial calcium uniporter, were applied during the first $10 \mathrm{~min}$ of the sustained reperfusion.

3) Infarct size measurement: Infarct size was determined by the 2,3,5-triphenyltetrazolium chloride (TTC) staining method. At the end of $120 \mathrm{~min}$ reperfusion, the coronary artery was re-occluded, and after the heart was perfused with $1 \%$ Evans blue, it was frozen at $-20^{\circ} \mathrm{C}$ for $2-3$ hours. Then the heart was cut into slices and stained with $1 \%$ TTC. Infarct and risk areas were measured with planimetry using Image/J software from NIH. Infarct size was expressed as percentage of risk zone.

4) Lactate dehydrogenase measurement: Coronary effluent at 5, 10, 15, 20 and 30 min during reperfusion was collected and the lactate dehydrogenase (LDH) concentration was measured spectrophotometrically.

5) Statistical analysis: All values are expressed as mean \pm SD. Statistical significance was determined by oneway ANOVA with Newman-Keuls post-hoc test. Values of $\mathrm{p}<0.05$ were considered significant.

\section{RESULTS}

A. Role of mitochondrial calcium uniporter in myocardial injury induced by ischemia and reperfusion 
Administration of $20 \times 10^{-6} \mathrm{M}$ spermine during the first 10 min of reperfusion increased the LDH release throughout the reperfusion period (Fig. 1), but did not induce significant changes in infarct size (Fig. 2), LVDP, $+\mathrm{dP} / \mathrm{dtmax}$ or LVEDP (Fig. 3). Treatment with ruthenium red $\left(5 \times 10^{-6} \mathrm{M}\right)$ during the first $10 \mathrm{~min}$ of reperfusion decreased the infarct size (Fig. 2) and the LDH release during reperfusion (Fig. 1), which were associated with augmentation of LVDP, +dP/dtmax and LVEDP recovery (Fig. 3).

\section{B. Relationship of ischemic preconditioning to the mitochondrial calcium uniporter}

In the IPC group, infarct size and LDH release were significantly less than those in the group with ischemia/reperfusion alone (Figs. 1 and 2), while LVDP, $+\mathrm{dP} / \mathrm{dtmax}$ and LVEDP were showed better recovery (Fig. $3)$. Treatment with spermine $\left(20 \times 10^{-6} \mathrm{M}\right)$ reduced the effect induced by IPC (Figs. 1, 2 and 3).

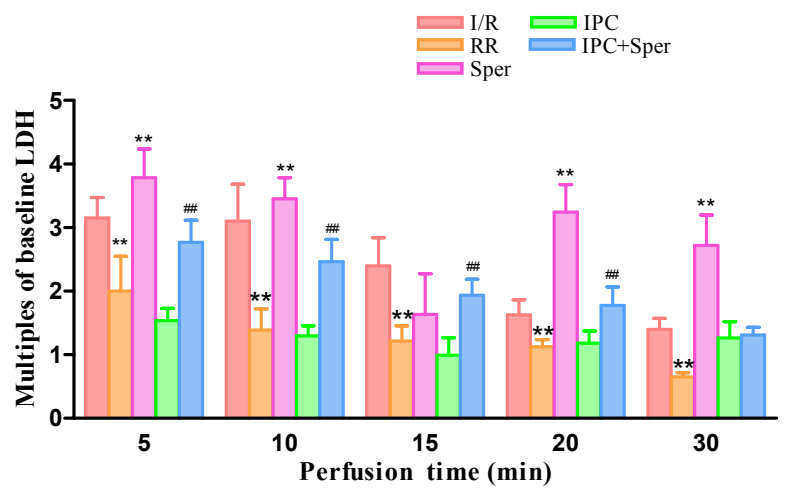

Fig. 1. Effects of ischemia/reperfusion (I/R), ruthenium red (RR, $5 \times 10^{-6} \mathrm{M}$ ), spermine (Sper, $20 \times 10^{-6} \mathrm{M}$ ) and ischemic preconditioning (IPC) on lactate dehydrogenase (LDH) release in isolated rat hearts. LDH expressed as multiples of pre-drug/preischemia baseline values. $\mathrm{n}=8$, for each group.

** $\mathrm{P}<0.01$ vs. $\mathrm{I} / \mathrm{R} ;{ }^{\#} \mathrm{P}<0.01$ vs. IPC

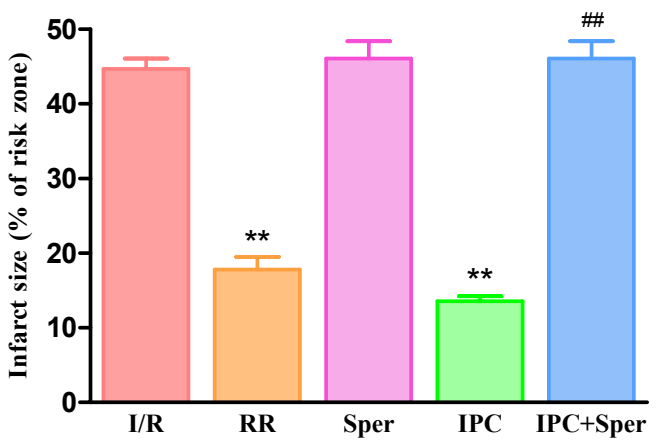

Fig. 2. Effects of ischemia/reperfusion (I/R), ruthenium red (RR, $5 \times 10^{-6} \mathrm{M}$ ), spermine (Sper, $20 \times 10^{-6} \mathrm{M}$ ) and ischemic preconditioning (IPC) on infarct size in isolated rat hearts. $\mathrm{n}=8$, for each group.

${ }^{* *} \mathrm{P}<0.01$ vs. I/R; ${ }^{\# \#} \mathrm{P}<0.01$ vs IPC
A



B

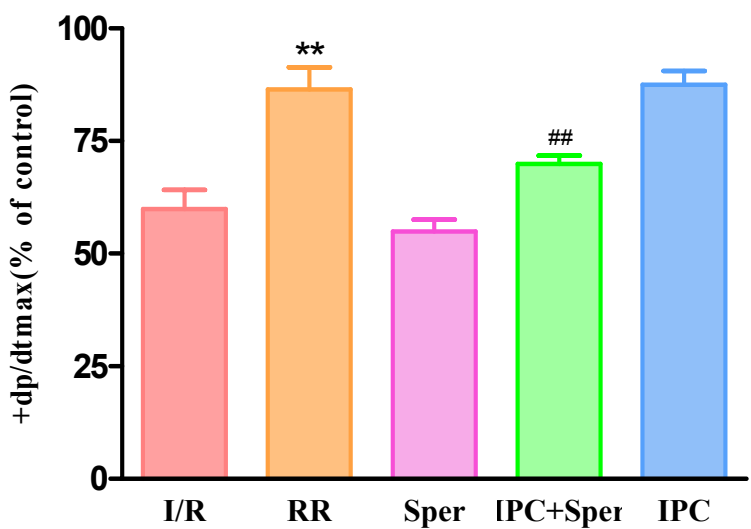

C

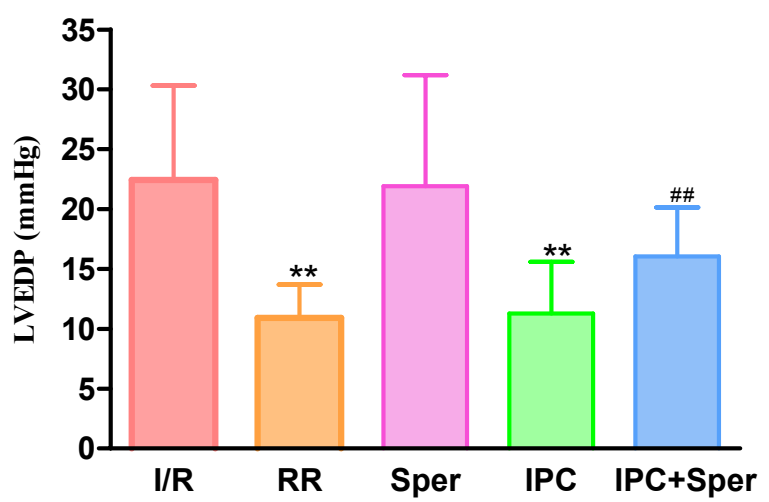

Fig. 3. Effects of ischemia/reperfusion (I/R), ruthenium red (RR, $5 \times 10^{-6} \mathrm{M}$ ), spermine (Sper, $20 \times 10^{-6} \mathrm{M}$ ) and ischemic preconditioning (IPC) on LVDP (A), $+\mathrm{dP} / \mathrm{dtmax}$ (B) and LVEDP (C) in isolated rat hearts. Data were collected at $30 \mathrm{~min}$ of reperfusion. $\mathrm{n}=8$, for each group.

$* * \mathrm{P}<0.01$ vs. $\mathrm{I} / \mathrm{R} ;{ }^{\#} \mathrm{P}<0.01$ vs. IPC 


\section{DISCUSSION}

In the present study, we found that ruthenium red, a blocker of the mitochondrial calcium uniporter, reduced, while spermine, an activator of the uniporter, aggravated ischemia/reperfusion-induced myocardial injury. Furthermore, the cardioprotective effect of IPC was attenuated by spermine, suggesting that the mitochondrial calcium uniporter is involved in the cardiac effects of IPC.

The calcium uniporter is one of the mechanisms by which mitochondria exchange calcium with the cytosol. The uniporter transports calcium from the cytosol into the intramitochondrial compartment and increases the calcium level in the mitochondrial matrix. The driving force for calcium into mitochondria via the uniporter depends on the electrochemical gradient across the mitochondrial membrane [7]. The mitochondrial membrane potential is 150 to $-180 \mathrm{mV}$, a gradient which facilitates calcium influx. Prolonged ischemia produces an increased calcium concentration in the cytosol, which results in mitochondrial accumulation of calcium during reperfusion, leading to mitochondrial calcium overload, myocardial damage and cell death $[8 ; 9]$. Therefore, preventing or reducing mitochondrial calcium accumulation by inhibiting the mitochondrial calcium uniporter might be expected to exert a beneficial effect on the heart during ischemia and reperfusion. This was confirmed in the present study, since treatment with the inhibitor ruthenium red antagonized the harmful effects of ischemia and reperfusion.

We extended these findings by testing the hypothesis that the mitochondrial calcium uniporter may also be involved in the cardioprotection by ischemic preconditioning. This was confirmed by our finding that the uniporter activator spermine canceled the protective effect of ischemic preconditioning, suggesting that it does play a role in this process. The uniporter is a highly calciumselective and inwardly rectifying ion channel, and the open probability of the channel is about $99 \%$ at $-200 \mathrm{mV}$ and declines to approximately $11 \%$ at $-80 \mathrm{mV}$ [10]. Opening mitochondrial ATP-sensitive potassium channels (mitoK $\mathrm{ATP}_{\mathrm{AT}}$ ) has been shown to depolarize the mitochondrial membrane potential and thus protect the heart during ischemia and reperfusion $[11 ; 12 ; 13]$. Thus, the mitochondrial calcium uniporter appears to be the target molecule of the intracellular signaling pathway between mitoK $\mathrm{KTP}_{\mathrm{ATP}}$ and cardioprotective end-effect; i.e. mitoK $_{\mathrm{ATP}}$ opening results in depolarization, which then closes the uniporter and thus prevents mitochondria from calcium overload and protects the myocardium from ischemia/reperfusion damage.

\section{CONCLUSION}

In summary, this study shows that inhibition of the mitochondrial uniporter during reperfusion protects isolated rat hearts subjected to ischemia and reperfusion. Cancellation of IPC-induced cardioprotection by activating the mitochondrial calcium uniporter reveals its potential role in this process.

\section{REFERENCES}

[1] Murry, C. E., Jennings, R. B., and Reimer, K. A., "Preconditioning with ischemia: a delay of lethal cell injury in ischemic myocardium," Circulation, vol. 74, no. 5, pp. 11241136, Nov.1986.

[2] Yellon, D. M., Baxter, G. F., Garcia-Dorado, D., Heusch, G., and Sumeray, M. S., "Ischaemic preconditioning: present position and future directions," Cardiovasc.Res., vol. 37, no. 1, pp. 21-33, Jan.1998.

[3] Allard, M. F., Flint, J. D., English, J. C., Henning, S. L., Salamanca, M. C., Kamimura, C. T., and English, D. R., "Calcium overload during reperfusion is accelerated in isolated hypertrophied rat hearts," J.Mol.Cell Cardiol., vol. 26, no. 12, pp. 1551-1563, Dec.1994.

[4] Meno, H. and Kanaide, H., "[Calcium overload and myocardial injury]," Tanpakushitsu Kakusan Koso, vol. 35, no. 10, pp. 1845-1853, July1990.

[5] Wang, L., Cherednichenko, G., Hernandez, L., Halow, J., Camacho, S. A., Figueredo, V., and Schaefer, S., "Preconditioning limits mitochondrial $\mathrm{Ca}(2+)$ during ischemia in rat hearts: role of K(ATP) channels," Am.J.Physiol Heart Circ.Physiol, vol. 280, no. 5, pp. H2321-H2328, May.2001.

[6] Kirichok, Y., Krapivinsky, G., and Clapham, D. E., "The mitochondrial calcium uniporter is a highly selective ion channel," Nature, vol. 427, no. 6972, pp. 360-364, Jan.2004.

[7] Gunter, T. E. and Pfeiffer, D. R., "Mechanisms by which mitochondria transport calcium," Am.J.Physiol, vol. 258, no. 5 Pt 1, pp. C755-C786, May.1990.

[8] Buja, L. M., "Modulation of the myocardial response to ischemia," Lab Invest, vol. 78, no. 11, pp. 1345-1373, Nov.1998.

[9] Di Lisa, F. and Bernardi, P., "Mitochondrial function as a determinant of recovery or death in cell response to injury," Mol.Cell Biochem., vol. 184, no. 1-2, pp. 379-391, July1998.

[10] Kirichok, Y., Krapivinsky, G., and Clapham, D. E., "The mitochondrial calcium uniporter is a highly selective ion channel," Nature, vol. 427, no. 6972, pp. 360-364, Jan.2004.

[11] Gross, G. J. and Peart, J. N., "KATP channels and myocardial preconditioning: an update," Am.J.Physiol Heart Circ.Physiol, vol. 285, no. 3, pp. H921-H930, Sept.2003.

[12] Ala-Rami, A., Ylitalo, K. V., and Hassinen, I. E., "Ischaemic preconditioning and a mitochondrial KATP channel opener both produce cardioprotection accompanied by F1F0-ATPase inhibition in early ischaemia," Basic Res.Cardiol., vol. 98, no. 4 pp. 250-258, July.2003.

[13] Holmuhamedov, E. L., Wang, L., and Terzic, A., "ATPsensitive $\mathrm{K}+$ channel openers prevent $\mathrm{Ca} 2+$ overload in rat cardiac mitochondria," J.Physiol, vol. 519 Pt 2 pp. 347-360, Sept.1999. 